\title{
Sex- and Age-Related Differences in Bone Microarchitecture in Men Relative to Women Assessed by High-Resolution Peripheral Quantitative Computed Tomography
}

\author{
Shreyasee Amin ${ }^{1,2}$ and Sundeep Khosla ${ }^{3}$ \\ ${ }^{1}$ Division of Rheumatology, Department of Internal Medicine, College of Medicine, Mayo Clinic, 200 First Street SW, Rochester, \\ MN 55905, USA \\ ${ }^{2}$ Division of Endocrinology, Diabetes, Metabolism and Nutrition, Department of Internal Medicine, College of Medicine, \\ Mayo Clinic, 200 First Street SW, Rochester, MN 55905, USA \\ ${ }^{3}$ Division of Epidemiology, Department of Health Sciences Research, College of Medicine, Mayo Clinic, 200 First Street SW, \\ Rochester, MN 55905, USA
}

Correspondence should be addressed to Shreyasee Amin, amin.shreyasee@mayo.edu

Received 5 September 2011; Accepted 31 October 2011

Academic Editor: Pawel Szulc

Copyright (C) 2012 S. Amin and S. Khosla. This is an open access article distributed under the Creative Commons Attribution License, which permits unrestricted use, distribution, and reproduction in any medium, provided the original work is properly cited.

\begin{abstract}
The trabecular and cortical compartments of bone each contributes to bone strength. Until recently, assessment of trabecular and cortical microstructure has required a bone biopsy. Now, trabecular and cortical microstructure of peripheral bone sites can be determined noninvasively using high-resolution peripheral quantitative computed tomography (HR-pQCT). Studies that have used HR-pQCT to evaluate cohorts of both men and women have provided novel insights into the changes in bone microarchitecture that occur with age between the sexes, which may help to explain the lower fracture incidence in older men relative to women. This review will highlight observations from these studies on both the sex- and age-related differences in trabecular and cortical microstructure that may underlie the differences in bone strength, and thereby fracture risk, between men and women.
\end{abstract}

\section{Introduction}

High-resolution peripheral quantitative computed tomography (HR-pQCT) is a novel imaging modality that noninvasively assesses trabecular and cortical bone microstructure at the distal radius and tibia $[1,2]$. Although areal bone mineral density (aBMD), as assessed by dual-energy Xray absorptiometry (DXA), remains an important predictor of fractures, DXA technology is nevertheless limited in its inability to measure the trabecular and cortical bone compartments separately, each of which contributes to bone strength [3]. The ability to assess these compartments may help improve fracture prediction in men and women. Until recently, assessment of trabecular and cortical microstructure has required a bone biopsy. With the development and subsequent validation of HR-pQCT [1,4-7], trabecular and cortical microstructure of peripheral bone sites can now be determined noninvasively and is thus more amenable for clinical study. Indeed, there are a growing number of studies that have now used HR-pQCT to evaluate relatively large cohorts of men and women. The findings from these studies have provided important advances to our understanding of the age-related changes in bone microarchitecture that occur, which may in turn help to explain the lower fracture incidence observed in aging men relative to aging women.

\section{High-Resolution Peripheral Quantitative Computed Tomography}

2.1. HR-pQCT Imaging and Processing. Bone histomorphometry from iliac crest bone biopsies provided invaluable two-dimensional information on bone microarchitecture [9], but the use of bone biopsies in clinical studies was 


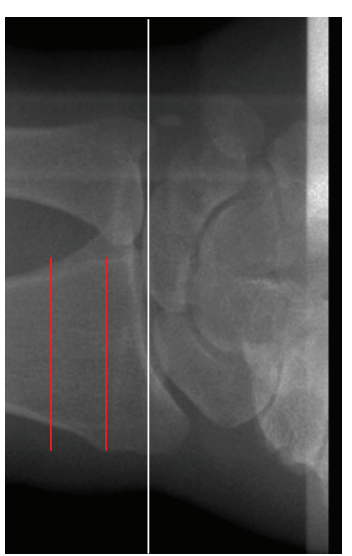

(a)
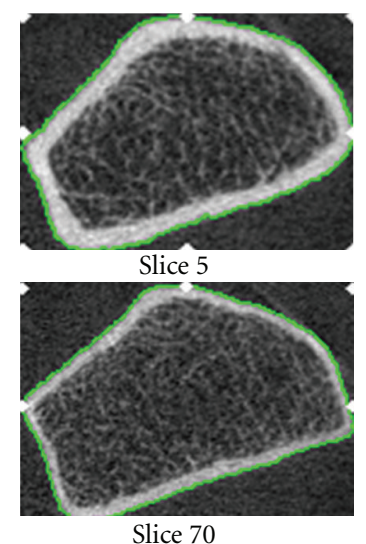
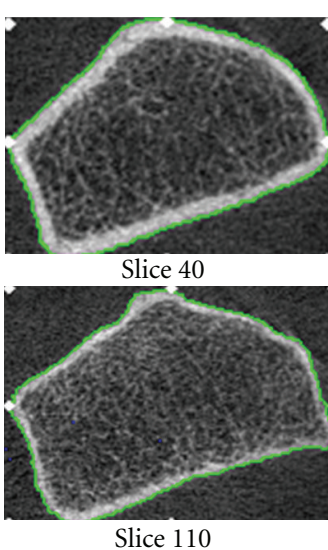

(b)

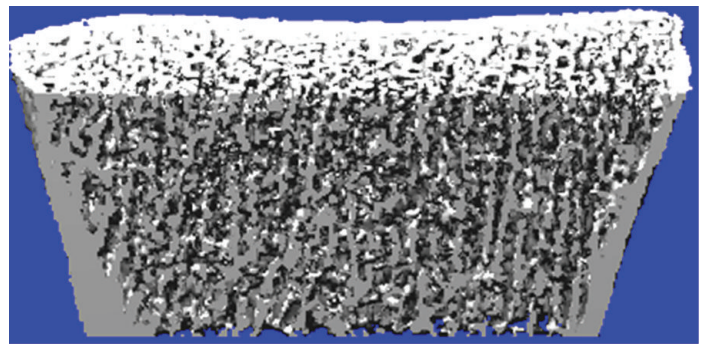

(c)

FIGURE 1: (a) Radiograph showing the site of imaging by HR-pQCT at the distal radius. The white line indicated the proximal level of the joint space, and the red lines indicate the section of bone over which images are acquired. (b) Representative cross-sectional images from the stack of CT slices. (c) Representative 3D image. Images were obtained using the initial prototype of the HR-pQCT scanner (Figure is reprinted with permission from Khosla et al. [8] ${ }^{\circledR} 2006$ American Society for Bone and Mineral Research).

limited due to its invasive nature and the inability to assess a section of bone more than once. Furthermore, the threedimensional (3D) aspect of bone microarchitecture could not be taken into account to fully understand its biomechanical properties. Imaging modalities were thus developed that could assess, noninvasively, the 3D bone microarchitecture in vivo $[1,2]$ and this work led to the now commercially available 3D HR-pQCT scanner (Xtreme CT, Scanco Medical AG, Switzerland; voxel size $82 \mu \mathrm{m}$ ) that can image the distal radius and distal tibia of human subjects. The imaging protocol provides not only an assessment of both trabecular and cortical bone microstructure parameters, which will be described in more detail below, but also total volumetric BMD (vBMD, $\mathrm{g} / \mathrm{cm}^{3}$ ) of the distal radius or tibia as well as separate measures for trabecular and cortical vBMD. In recent software updates, an assessment of cortical porosity has become available [10] as well as an assessment of bone strength at the radius and tibia determined through microfinite element analysis (IPL v1.12, Scanco Medical AG) although similar parameters can be derived from the images independently from the built-in software [11]. Several additional trabecular and cortical microstructure parameters are provided through the commercial software; however, these novel parameters have not been as well studied as those described below.

Imaging of the distal radius or tibia by HR-pQCT involves a similar protocol with the acquisition of a 3D stack of 110 high-resolution CT slices at either the distal end of the radius (Figure 1(a)) or tibia, with an isotropic voxel size and slice thickness of $82 \mu \mathrm{m}$. The processing and analyses of these images has been validated [4-7]. Figures 1(b) and 1 (c) show representative cross-sectional scan slices and 3D images, respectively, that can be obtained from HR-pQCT imaging.

2.2. Assessment of Trabecular Parameters by HR-pQCT. Some trabecular microstructure parameters assessed using HRpQCT are measured directly while others are derived. The trabecular vBMD is determined directly, and then the trabecular bone volume/total volume (BV/TV) is derived, assuming a mineral density of fully mineralized bone of $1.2 \mathrm{~g}$ hydroxyapatite $/ \mathrm{cm}^{3}$. Recognizing that individual trabeculae would not be resolved at their correct thickness $(\sim 100 \mu \mathrm{m})$ due to partial volume effects, a thickness-independent structure extraction is employed whereby $3 \mathrm{D}$ ridges (the center points of the trabeculae) are detected in the graylevel images [5]. Trabecular number $(\mathrm{TbN}, 1 / \mathrm{mm})$ is then taken as the inverse of the mean spacing of the ridges [6]. Then, in analogy with standard histomorphometry [12], trabecular thickness ( $\mathrm{TbTh}, \mathrm{mm})$ is calculated using the formula $\mathrm{TbTh}=\mathrm{BV} / \mathrm{TV} \div \mathrm{TbN}$, and trabecular spacing or separation (TbSp, mm) is calculated using the formula TbSp $=(1-\mathrm{BV} / \mathrm{TV}) \div \mathrm{TbN}$. Validation studies show excellent correlation $(R \geq 0.96, P<0.0001)$ for these parameters when 
compared with the gold-standard ex vivo microcomputed tomography $(\mu \mathrm{CT})$ technique [13].

2.3. Assessment of Cortical Parameters by HRp-QCT. The cortex is segmented from the gray scale image with a Gaussian filter and threshold [6]. Cortical vBMD $\left(\mathrm{g} / \mathrm{cm}^{3}\right)$ bone cross-sectional area $\left(\mathrm{CSA}, \mathrm{mm}^{2}\right)$ and cortical crosssectional area $\left(\mathrm{Ct} C S A, \mathrm{~mm}^{2}\right)$ are measured directly, and the periosteal circumference calculated from the contour. Cortical thickness (CtTh, $\mathrm{mm}$ ) is then derived using the formula $\mathrm{CtTh}=\mathrm{Ct} \mathrm{CSA} \div$ circumference. Excellent correlation has been shown for CtTh measurements with HR-pQCT versus $\mu \mathrm{CT}[7]$.

\section{Bone Microarchitecture by HR-pQCT in Men}

Recent studies that have assessed both men and women using HR-pQCT have led to important insights into the sex- and age-related differences in bone microarchitecture. Greater bone strength in men than women is, in part, related to larger bone size of men. However, differences between the sexes with respect to both the trabecular and cortical compartments of bone may also contribute to their differences in bone strength and fracture risk.

\subsection{Differences in Bone Microarchitecture in Young Men versus} Young Women. Khosla et al. [8] first reported on the bone microarchitecture by HR-pQCT in both men and women. Using the initial prototype of the HR-pQCT scanner, the distal radius was imaged in 278 men and 324 women (ages of 21-97 years), who represented an age-stratified, random sample of the Rochester, MN community adults [8]. Relative to young women age $20-29$ years of age $(n=17)$, young men of a similar age range $(n=19)$ had higher BV/TV and greater TbTh but had similar TbN and TbSp [8]. Young men also had greater bone CSA, but similar CtTh, when compared with young women [8].

Sode et al. [14] imaged both the distal radius and tibia in 146 healthy volunteers ( 53 men and 93 women), aged 20-78 years. They found that young men (age 20-29 years) had greater BV/TV and TbTh at both the distal radius and tibia when compared with young women of the same age range [14]. TbN and TbSp were not significantly different between the sexes at the radius but were at the tibia [14]. Findings were thus similar to what was observed by Khosla et al. at the radius. The tibia was not imaged in the study by Khosla et al., but findings by Sode et al. suggested that the sex-related differences in bone microarchitecture may not be the same between the radius and tibia sites. Sode et al. also noted substantial variations in trabecular parameters within a cross-section of either the distal radius or tibia and identified sex-specific subregional variations in trabecular microstructure [14].

In a population-based study from Canada involving 202 men and 442 women (ages 20-99 years) where both the distal radius and tibia were measured, MacDonald et al. [15] reported that young men (age 20-29 years, $N=28$ ) had greater bone size, $\mathrm{BV} / \mathrm{TV}$ and $\mathrm{TbTh}$ at the distal radius when compared with young women of the same age range $(N=$ 58), observations that were again similar to reports at the same site by Khosla et al. and Sode et al. In contrast, they observed that young men also had greater $\mathrm{TbN}$ and $\mathrm{CtTh}$ than women [15]. At the distal tibia, young men again had greater bone size, BV/TV, TbN and CtTh than women [15]. $\mathrm{TbTh}$ at the tibia was also slightly higher in men but did not reach statistical significance [15].

Interestingly, in a study involving almost 300 adolescent boys and girls between the ages of 15-20 years who had their distal tibia imaged by HR-pQCT, sex-related differences were more apparent by the age of 17-20 years, with boys in this age range having greater bone size, BV/TV and CtTh relative to girls [16]. However in this study, TbTh was not significantly different between the sexes at any age, whereas $\mathrm{TbN}$ was higher, while TbSp was lower, in boys than girls at all ages [16]. However, a cross-sectional study by Wang et al., involving almost 130 boys and girls ages 5-18 years, found that BV/TV and TbTh increased more in boys than girls over puberty at both the radius and tibia sites, while TbN remained similar for both boys and girls [17]. Similarly, in a cross-sectional study by Kirmani et al. assessing the distal radius of 140 healthy boys and girls ages 6-21 years, BV/TV and $\mathrm{TbTh}$ also increased more in boys than girls over puberty [11]. TbN transiently increased in boys more than girls across age but then became similar again in both sexes at more mature ages [11].

Collectively, these studies confirm that there are clear bone microarchitectural differences apparent between young men and women, particularly bone size, BV/TV and TbTh, with these differences developing over puberty and appearing to be established as early as late adolescence. These studies also suggest that sex-related differences for some parameters of bone microstructure may be site-specific. Nevertheless, there was a lack of consistency among studies on findings for some parameters, but they may reflect imaging resolution issues or power limitations.

\subsection{Age-Related Differences in Bone Microarchitecture in Men.} To date, studies reporting on the age-related differences in bone microarchitecture in men and women have been crosssectional in nature. The determination of true age-related changes in bone microarchitecture will require longitudinal studies. Nevertheless, these cross-sectional studies do provide key information on differences in the trabecular and cortical compartments of bone between young and old and thus impart the most practical insights on structural changes that may be related to aging in men and women.

In the study by Khosla et al., cross-sectional decreases in $\mathrm{BV} / \mathrm{TV}$ at the radius between the ages of 20 and 90 years were reported to be similar in men and women [8]. However, in contrast to women, where $\mathrm{TbN}$ decreased and TbSp increased with age, there were little age-related changes in these parameters in men [8]. On the other hand, TbTh appeared to decrease to a greater extent in men than women $(P<0.01)$ [8] (Figure 2). These findings suggested that the mechanism for loss in BV/TV with aging in men and women may be different, with loss of trabeculae being the main factor in women but trabecular thinning being the 


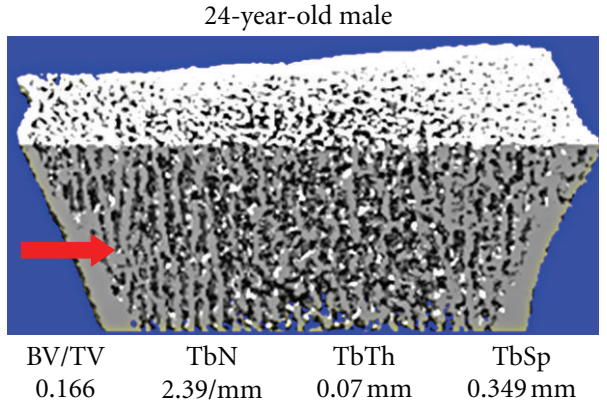

(a)

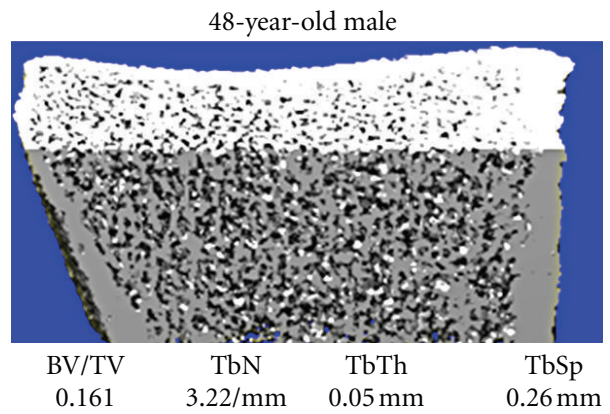

(c)

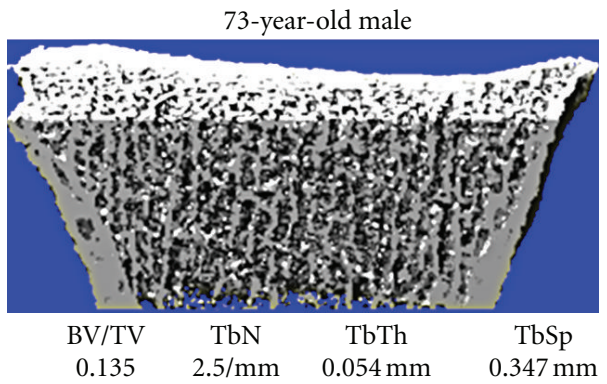

(e)

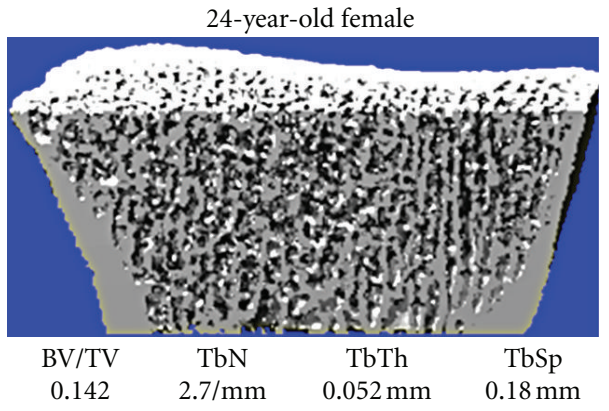

(b)

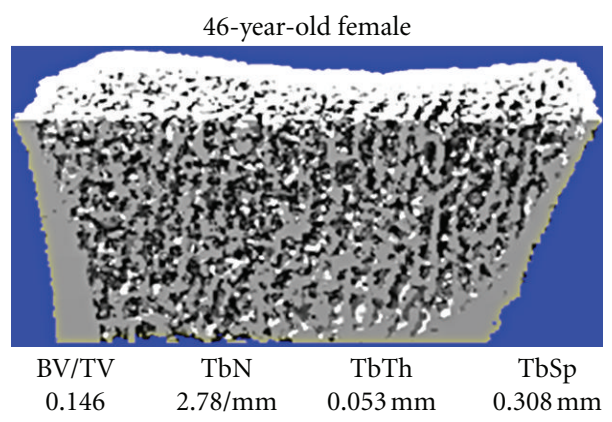

(d)

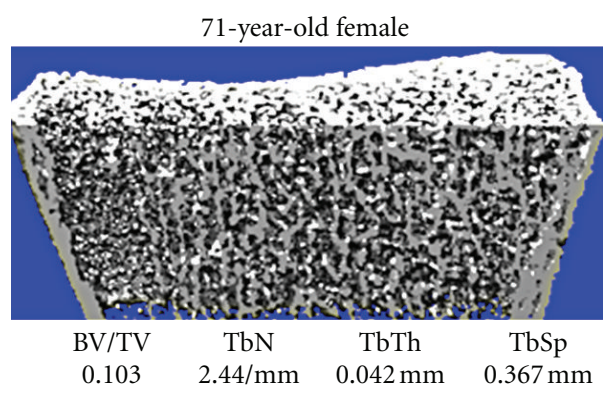

(f)

FIGURE 2: Representative reconstructions of high-resolution peripheral quantitative computed tomography images from young, middleaged, and elderly men (left) and women (right). Values for trabecular microstructural variables for each subject are also provided. The arrow indicates the prominent plate-like trabeculae in the young man, which are less prominent or absent in the other images. Images were obtained using the initial prototype of the HR-pQCT scanner. (Figure is reprinted with permission from Khosla et al. [8] ${ }^{\circledR} 2006$ American Society for Bone and Mineral Research).

mechanism for men. As decreases in TbN have been shown to have greater impact on impairing bone strength compared with decreases in TbTh [18], it was suggested that these findings could explain the lower life-long fracture risk in men relative to women. MacDonald et al. observed similar findings as Khosla et al. at the distal radius [15]. However, at the distal tibia, MacDonald et al. found that TbTh, TbN, and TbSp changed to a similar extent in both men and women with age [15]. The distal tibia was not assessed in the study by Khosla et al. Patterns of trabecular loss may thus vary by skeletal site. Sode et al. reported that age-related differences in men and women were more prominent at particular subregions of each bone [14]. On the other hand, Dalzell et al. failed to see any significant association between trabecular microstructure parameters and age, at either the distal radius or tibia, in their study involving 58 men and 74 women (ages 20-79 years) from the UK [19].

Khosla et al. reported that, in both men and women, bone CSA increased with aging, but while the percentage change was similar between men and women, older men continued to have greater bone size than older women [8]. In contrast, CtTh decreased to a lesser extent in men than women $(-38 \%$ versus $-52 \%$, resp.; $P=0.001)$ [8]. The greater bone size and thicker cortex thus confers a greater biomechanical advantage for bones of elderly men compared with elderly women [8]. In contrast to the trabecular compartment, where parameters showed cross-sectional agerelated declines before and after age 50 years, the cortical parameters tended to remain stable until after age 50 years when they then started to decline [8]. Dalzell et al. also 
identified that cortical thickness declined faster in women than men after age 50 [19].

MacDonald et al. observed that cortical porosity increased to a lesser extent in men than women with advancing age [15]. However, cortical porosity appeared to be greater in men than women at all age ranges except at the eighth decade [15]. In a study involving 57 men and 94 women (age range 20-78 years), Burghardt et al. [20] reported similar observations, with men having greater cortical porosity than women, especially before age 30 . Differences became attenuated in those over the age of 50 years due to a 3 -fold increase in cortical porosity in women from age 20 to 60 years, with men having an increase in cortical porosity of only $60 \%$ over the same age range [20].

Based on observations from these cross-sectional studies, men appear to have more trabecular thinning than dropout with increasing age, while women have both trabecular thinning and dropout. Furthermore, men have greater bone size than women across age and suffer less cortical thinning than women with aging. Although men have greater cortical porosity than women, it increases more in women than men with age. Overall, these apparent age-related changes in trabecular and cortical microstructure in men would thus seem to confer less of an adverse effect on bone strength and, thereby, explain the lower fracture risk, in aging men when compared with aging women.

\section{Bone Microarchitecture by HR-pQCT and Fracture Risk in Men}

To date, only the Structure of the Aging Men's Bones (STRAMBO) study has evaluated the relation between prevalent fractures in men with bone microarchitecture parameters from HR-pQCT [21, 22]. In a subset of men from the STRAMBO cohort, 185 men (mean age, 71 years) with a prevalent fracture and 185 men without fracture had both the distal radius and tibia imaged by HR-pQCT and had assessments of bone strength by finite element analysis [21]. At both bone sites, $\mathrm{CtTh}, \mathrm{TbN}$, and $\mathrm{TbSp}$ were significantly worse in cases than controls, as was the aBMD and vBMD [21]. Bone strength parameters of stiffness and failure load also were $8-9 \%$ lower in fracture cases relative to controls $(P<0.01)$ [21]. In a larger cohort from STRAMBO, involving 920 men over the age of 50 years who had HRpQCT measurements at the distal radius and tibia, 98 men had a prevalent vertebral fracture, and 100 men had a prevalent peripheral fracture [22]. Again, almost all bone microarchitecture parameters from HRpQCT scans were worse in men with either vertebral or peripheral fractures [22]. Following adjustment for aBMD, there were no associations observed between bone microarchitecture parameters and peripheral fractures, whereas cortical vBMD and $\mathrm{Ct} \mathrm{Th}$ at both the distal radius and tibia remained lower in men with vertebral fractures [22]. Whether the bone microarchitecture parameters based on the radius or tibia will be able to predict overall or site-specific fragility fractures in men prospectively remains to be determined.
Imaging studies using HR-pQCT of bone microarchitecture among children at various stages of puberty also have provided further understanding on the pathogenesis of increased fractures in adolescence among both boys and girls although findings are again limited by their crosssectional nature. In the study by Kirmani et al. [11], the proportion of load borne by cortical bone and the ratio of cortical to trabecular bone volume at the radius decreased transiently during mid-to-late puberty in both sexes, with apparent cortical porosity peaking during this time [11]. These findings appeared to mirror the incidence of distal forearm fractures seen in boys and girls of this age group [11]. Regional deficits in cortical bone were thus suggested to underlie the adolescent peak in forearm fractures [11], a hypothesis that was also suggested by Wang et al. [17].

\section{Conclusion}

Although studies in men imaged by HR-pQCT are limited, current findings have provided novel insights into the sex- and age-related differences in trabecular and cortical microstructure that underlie the differences in bone strength and fracture risk observed in men relative to women. Nevertheless, the cross-sectional nature of studies to date does limit the ability to make definitive conclusions, and longitudinal studies remain the ideal in order to best determine agerelated changes in bone microarchitecture and their relation to fracture risk in both men and women.

\section{Conflict of Interests}

There are no conflict of interests with respect to this work. Dr. Amin serves on a scientific advisory board for Merck \& Co, Inc.

\section{Acknowledgment}

This work was supported, in part, by research Grants AR027065 and AG004875 from the National Institutes of Health, U.S. Public Health Service.

\section{References}

[1] R. Muller, T. Hildebrand, and P. Ruegsegger, "Non-invasive bone biopsy: a new method to analyse and display the threedimensional structure of trabecular bone," Physics in Medicine and Biology, vol. 39, no. 1, pp. 145-164, 1994.

[2] D. Ulrich, B. Rietbergen, A. Laib, and P. Ruegsegger, "Mechanical analysis of bone and its microarchitecture based on in vivo voxel images," Technology and Health Care, vol. 6, no. 5-6, pp. 421-427, 1998.

[3] E. Seeman and P. D. Delmas, "Bone quality-the material and structural basis of bone strength and fragility," New England Journal of Medicine, vol. 354, no. 21, pp. 2212-2261, 2006.

[4] R. Müller, T. Hildebrand, H. J. Häuselmann, and P. Rüegsegger, "In vivo reproducibility of three-dimensional structural properties of noninvasive bone biopsies using 3DpQCT," Journal of Bone and Mineral Research, vol. 11, no. 11, pp. 1745-1750, 1996. 
[5] A. Laib, "Ridge number density: a new parameter for in vivo bone structure analysis," Bone, vol. 21, no. 6, pp. 541-546, 1997.

[6] A. Laib, H. J. Hauselmann, and P. Ruegsegger, "In vivo high resolution 3D-QCT of the human forearm," Technology and Health Care, vol. 6, no. 5-6, pp. 329-337, 1998.

[7] J. A. MacNeil and S. K. Boyd, "Accuracy of high-resolution peripheral quantitative computed tomography for measurement of bone quality," Medical Engineering and Physics, vol. 29, no. 10, pp. 1096-1105, 2007.

[8] S. Khosla, B. L. Riggs, E. J. Atkinson et al., "Effects of sex and age on bone microstructure at the ultradistal radius: a population-based noninvasive in vivo assessment," Journal of Bone and Mineral Research, vol. 21, no. 1, pp. 124-131, 2006.

[9] A. M. Parfitt, C. H. E. Mathews, and A. B. Villanueva, "Relationships between surface, volume, and thickness of iliac trabecular bone in aging and in osteoporosis. Implications for the microanatomic and cellular mechanisms of bone loss," Journal of Clinical Investigation, vol. 72, no. 4, pp. 1396-1409, 1983.

[10] A. J. Burghardt, H. R. Buie, A. Laib, S. Majumdar, and S. K. Boyd, "Reproducibility of direct quantitative measures of cortical bone microarchitecture of the distal radius and tibia by HR-pQCT," Bone, vol. 47, no. 3, pp. 519-528, 2010.

[11] S. Kirmani, D. Christen, G. H. Van Lenthe et al., "Bone structure at the distal radius during adolescent growth," Journal of Bone and Mineral Research, vol. 24, no. 6, pp. 10331042, 2009.

[12] A. M. Parfitt, M. K. Drezner, F. H. Glorieux et al., "Bone histomorphometry: standardization of nomenclature, symbols, and units. Report of the ASBMR Histomorphometry Nomenclature Committee," Journal of Bone and Mineral Research, vol. 2, no. 6, pp. 595-610, 1987.

[13] A. Laib and P. Rüegsegger, "Calibration of trabecular bone structure measurements of in vivo three-dimensional peripheral quantitative computed tomography with $28-\mu \mathrm{m}$ resolution microcomputed tomography," Bone, vol. 24 , no. 1 , pp. 35-39, 1999.

[14] M. Sode, A. J. Burghardt, G. J. Kazakia, T. M. Link, and S. Majumdar, "Regional variations of gender-specific and agerelated differences in trabecular bone structure of the distal radius and tibia," Bone, vol. 46, no. 6, pp. 1652-1660, 2010.

[15] H. M. MacDonald, K. K. Nishiyama, J. Kang, D. A. Hanley, and S. K. Boyd, "Age-related patterns of trabecular and cortical bone loss differ between sexes and skeletal sites: a populationbased HR-pQCT study," Journal of Bone and Mineral Research, vol. 26, no. 1, pp. 50-62, 2011.

[16] M. Burrows, D. Liu, S. Moore, and H. McKay, "Bone microstructure at the distal tibia provides a strength advantage to males in late puberty: an HR-pQCT study," Journal of Bone and Mineral Research, vol. 25, no. 6, pp. 1423-1432, 2010.

[17] Q. Wang, X. F. Wang, S. Iuliano-Burns, A. Ghasem-Zadeh, R. Zebaze, and E. Seeman, "Rapid growth produces transient cortical weakness: a risk factor for metaphyseal fractures during puberty," Journal of Bone and Mineral Research, vol. 25, no. 7, pp. 1521-1526, 2010.

[18] M. J. Silva and L. J. Gibson, "Modeling the mechanical behavior of vertebral trabecular bone: effects of age-related changes in microstructure," Bone, vol. 21, no. 2, pp. 191-199, 1997.

[19] N. Dalzell, S. Kaptoge, N. Morris et al., "Bone microarchitecture and determinants of strength in the radius and tibia: age-related changes in a population-based study of normal adults measured with high-resolution pQCT," Osteoporosis International, vol. 20, no. 10, pp. 1683-1694, 2009.
[20] A. J. Burghardt, G. J. Kazakia, S. Ramachandran, T. M. Link, and S. Majumdar, "Age- and gender-related differences in the geometric properties and biomechanical significance of intracortical porosity in the distal radius and tibia," Journal of Bone and Mineral Research, vol. 25, no. 5, pp. 983-993, 2010.

[21] N. Vilayphiou, S. Boutroy, P. Szulc et al., "Finite element analysis performed on radius and tibia HR-pQCT images and fragility fractures at all sites in men," Journal of Bone and Mineral Research, vol. 26, no. 5, pp. 965-973, 2011.

[22] P. Szulc, S. Boutroy, N. Vilayphiou, A. Chaitou, P. D. Delmas, and R. Chapurlat, "Cross-sectional analysis of the association between fragility fractures and bone microarchitecture in older men: the STRAMBO study," Journal of Bone and Mineral Research, vol. 26, no. 6, pp. 1358-1367, 2011. 


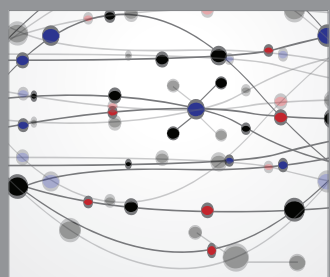

The Scientific World Journal
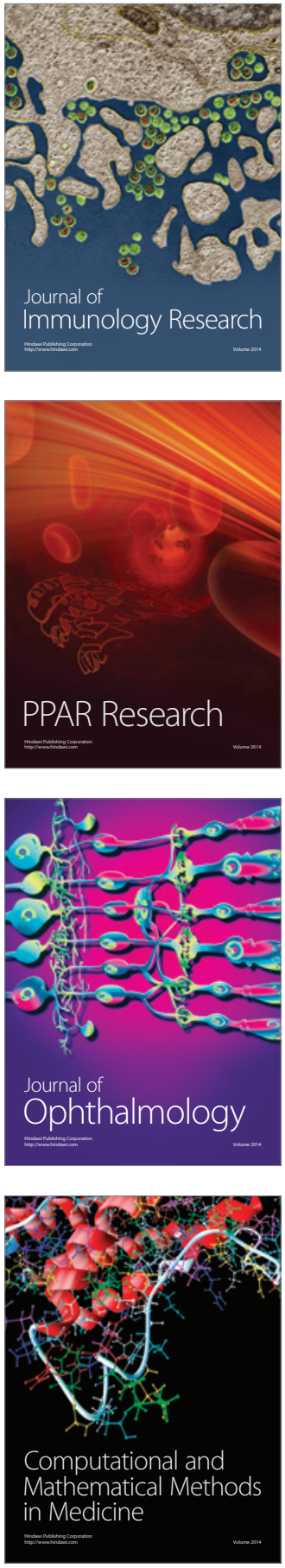

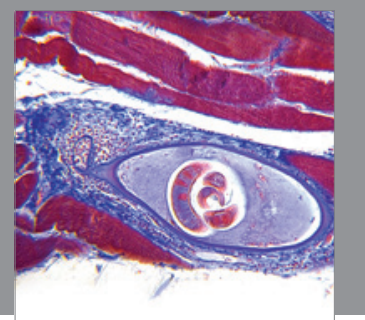

Gastroenterology

Research and Practice
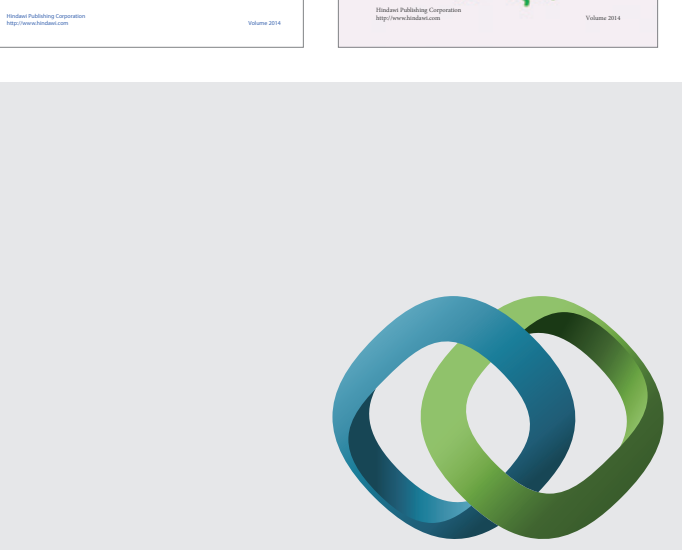

\section{Hindawi}

Submit your manuscripts at

http://www.hindawi.com
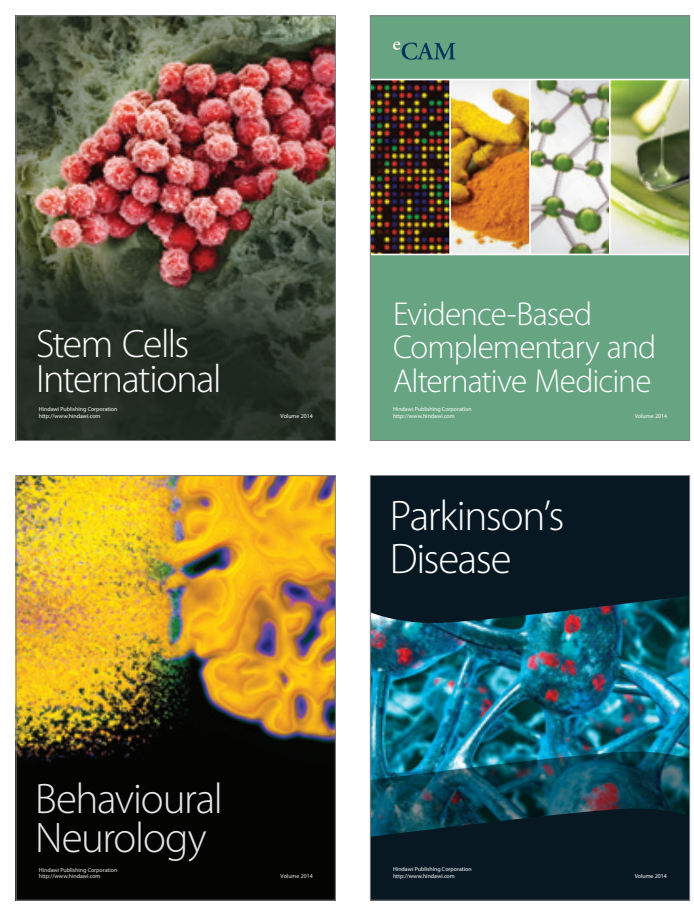

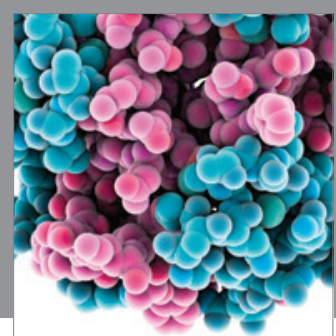

Journal of
Diabetes Research

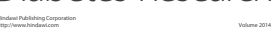

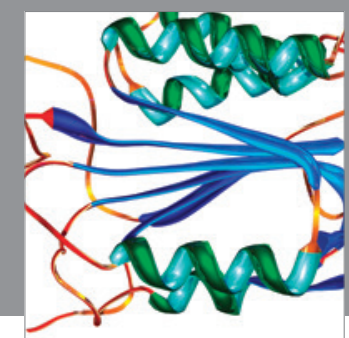

Disease Markers
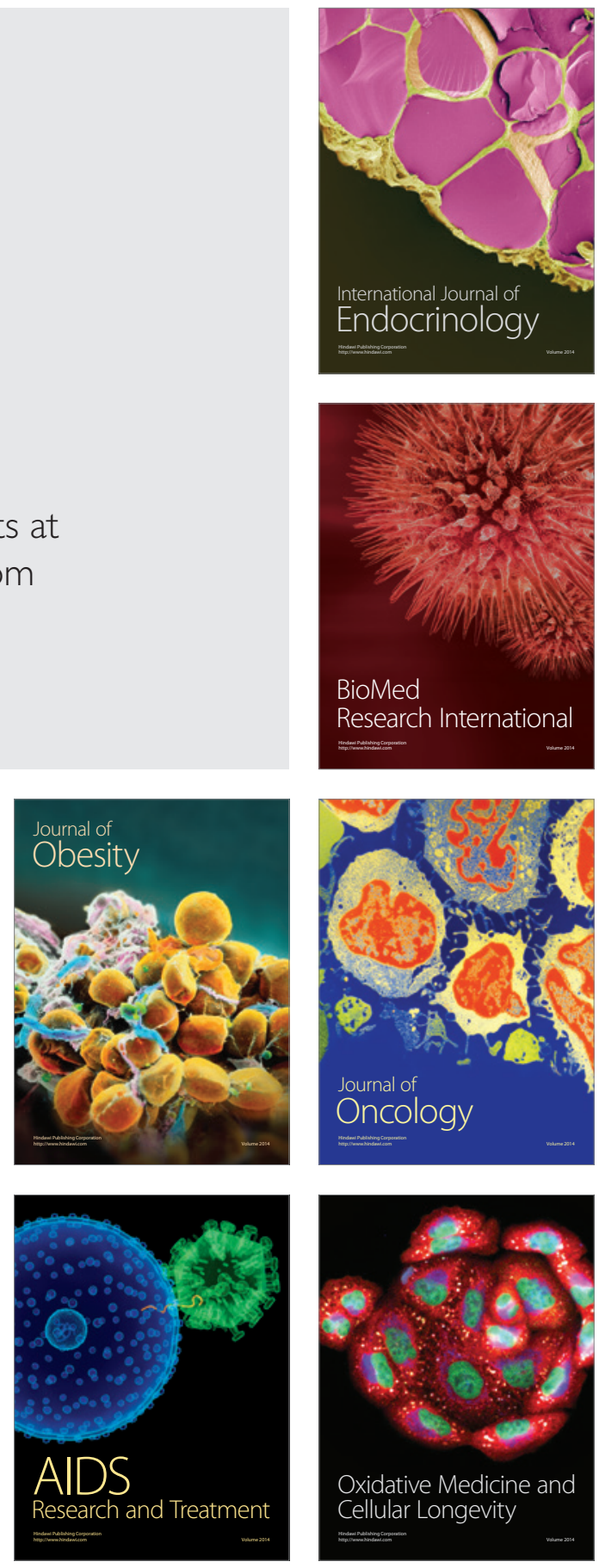